\title{
Measuring Space Deformation via Graphene under Constraints
}

\author{
Ahmed Jellal ${ }^{*}$ \\ Laboratory of Theoretical Physics, Faculty of Sciences, Chouaïb Doukkali University, \\ PO Box 20, 24000 El Jadida, Morocco \\ Canadian Quantum Research Center, 204-3002 32 Ave Vernon, \\ BC V1T 2L7, Canada
}

\begin{abstract}
We describe the lattice deformation in graphene under strain effect by considering the spacialmomenta coordinates do not commute. This later can be realized by introducing the star product to end up with a generalized Heisenberg algebra. Within such framework, we build a new model describing Dirac fermions interacting with an external source that noncommutative parameter $\kappa$ dependent. The solutions of the energy spectrum are showing effective Landau levels in similar way to the case of a real magnetic field applied to graphene. We show that some strain configurations would be able to explicitly evaluate $\kappa$ and then offer a piste toward its measurement.
\end{abstract}

PACS numbers: 71.15.-m, 02.40.Gh

Keywords: Graphene, noncommutative coordinates, Dirac equation, strain effect.

*a.jellal@ucd.ac.ma 


\section{Introduction}

Graphene was experimentally isolated in 2004 and is a sheet of carbon atoms arranged in hexagonal cells only a single atom thick [1]. It presents exostic properties in particular a linear dispersion relation, which is quite different to that of semi-conductors. This relation can be quantized under the influence of magnetic field applied to graphene sheet giving rise to the Landau levels [2]. Such field can be real or a manifestation of the graphene honeycomb under certain strain patterns generating the so-called pseudomagnetic field [3-5]. In fact, it was shown that the strained-induced pseudomagnetic field can easily reach quantizing values, exceeding $10 \mathrm{~T}$ in submicron devices for deformations less than $10 \%$ [6]. Besides the pseudomagnetic field can be generated in graphene through wafer-scale epitaxial growth or shallow triangular nanoprisms in the SiC substrate [7]. The importance of the quantized magnetic fields resides in the emergence of the anomalous quantum Hall effect [8,9]. Also it establishes a link with important theorems of modern mathematical physics as an example the existence of the zero-energy Landau level, which is a consequence of the Atiyah-Singer index theorem $[10,11]$.

On the other hand, the deformation based on the noncommutative geometry [12] is a power mathematical tool to deal with different issues in physics. Many works were reported on quantum mechanics in hopping to find some extensions and corrections to the known standard results. To this end, we may refer to our previous works on the quantum Hall effect [13] or statistical mechanics [14] as well other issues [15]. One way to invent the noncommutativity in a given physical system is to make use start product giving rise to a generalized Heisenberg algebra that involves commutation relations between positions and momenta coordinates in addition to the standard relations. Strictly speaking, noncommutativity can be imposed by treating the coordinates as commuting but requiring that composition of their functions is given in terms of the star product. In particular, it is shown that the noncommutative features affected the Landau levels by offering some corrections to the solutions of the energy spectrum either only taking the spatial-spatial being noncommute or both spatial-momenta do not commute, one may see [13-15] and references therein.

With respect to the extended Landau levels on the noncommutative plane, we propose a newly method to create a bridge between the noncommutativity of the coordinates and deformed graphene through the strain effect. To achieve this goal, we consider the Dirac fermions in pristine graphene moving in plane by taking spatial-momenta do not commute. The resulting Hamiltonian shows some similarities with that of the Landau problem for one particle experiencing a perpendicular magnetic field. This allows us to easily derive a quantized energy spectrum showing a dependency on the noncommutative parameter $\kappa$. To interpret our results, we offer two possibilities either by linking $\kappa$ to an effective magnetic field or expressing $\kappa$ in terms of the strain tensor components. To this end, we provide a way to measure $\kappa$ and fix its value by evaluating different physical parameters.

The present paper is organized as follows. In section 2, we build a theoretical model that captures the noncommutativity effect and leads to a Hamiltonian involving an effective vector potential taken in the symmetric gauge. The diagonalization of such Hamiltonian gives rise to effective Landau levels and eigenspinors similar to those of fermions in graphene subject to a perpendicular magnetic field. Naturally, these levels disappear and reduce to a linear dispersion relation by switching off $\kappa$. In section 3, we propose a method to shed light on the measurement of $\kappa$ by considering the strain tensor patterns in graphene and then offer numerical values. We conclude our results in the final section. 


\section{Building model}

Graphene can be described by a low-energy continuum model involving a matrix $4 \times 4$ because it possess two-Fermi points and each one has a two-fold band degeneracy. Such matrix has four-component envelope wavefunction whose components are labeled by a Fermi-point pseudospin $= \pm 1$ and a sublattice forming an honeycomb. Then one-pseudospin Hamiltonian for a free Dirac fermion reads as

$$
H=v_{\mathrm{F}} \vec{\sigma} \cdot \vec{p}
$$

where $\vec{\sigma}=\left(\sigma_{x}, \sigma_{y}\right)$ are the Pauli matrices, $\vec{p}=\left(p_{x}, p_{y}\right)$ is the momenta and $v_{\mathrm{F}} \approx 10^{6} \mathrm{~m} / \mathrm{s}$ is the Fermi velocity. One can easily show that the solution of the energy spectrum has a linear dispersion relation $E= \pm v_{\mathrm{F}} k$ with the wave vector $\vec{k}=\left(k_{x}, k_{y}\right)$. Now by applying a perpendicular magnetic $B$ to graphene sheet and choosing the symmetric gauge $\vec{A}_{B}=\frac{B}{2}(y,-x)$, the Hamiltonian (1) can be transformed to

$$
H_{B}=v_{\mathrm{F}}\left[\sigma_{x}\left(p_{x}+\frac{B e}{2} y\right)+\sigma_{y}\left(p_{y}-\frac{B e}{2} x\right)\right]
$$

having quantized energy $E_{n}= \pm v_{\mathrm{F}} \sqrt{2 B n}$ with $n$ is an integer value. In the next, we will apply a mathematical machinery based on the noncommutativity of spacial-momenta coordinates to obtain similar results to (2).

To deal with our task, we consider Dirac fermions living on the noncommutative space and impose the noncommutativity by treating the phase space coordinates as commuting but composing their functions with respect to the star product. Then, this process yields to the deformed Heisenberg algebra described by the commutation relations

$$
\left[x_{j}, p_{k}\right]=i \hbar \delta_{j k}, \quad\left[x_{j}, x_{k}\right]=i \theta \delta_{j k}, \quad\left[p_{j}, p_{k}\right]=i \kappa \delta_{j k}
$$

where $\theta$ and $\kappa$ are two antisymmetric noncommutative parameters measuring the noncommutativity between position $x_{i}$ and momenta $p_{i}$ coordinates. They play the same role as for the Planck constant in the canonical commutation relation between $x_{i}$ and $p_{i}$. Now taking into account of the noncommutativity, we can define an another Hamiltonian using the action of the Hamiltonian (1) via the start product on the spinor $\psi(x, y)$, namely

$$
H \star \psi(x, y)=H_{\kappa} \psi(x, y)
$$

which leads to the following mappings

$$
p_{x} \longrightarrow p_{x}+\frac{\kappa}{2 \hbar} y, \quad p_{y} \longrightarrow p_{y}-\frac{\kappa}{2 \hbar} x
$$

and consequently we end up with the noncommutative Dirac Hamiltonian

$$
H_{\kappa}=v_{\mathrm{F}}\left[\sigma_{x}\left(p_{x}+\frac{\kappa}{2 \hbar} y\right)+\sigma_{y}\left(p_{y}-\frac{\kappa}{2 \hbar} x\right)\right]
$$

At this level, we have some comments in order. Indeed, (6) looks like the Hamiltonian (2) for a real magnetic field associated to the symmetric gauge. This suggest to define an effective gauge $\overrightarrow{A_{\kappa}}=\frac{\kappa}{2 \hbar}(y,-x)$ and then write $H_{\kappa}$ in the compact form

$$
H_{\kappa}=v_{\mathrm{F}} \vec{\sigma} \cdot\left(\vec{p}+e \vec{A}_{\kappa}\right)
$$


and therefore by analogy to the relation $\vec{B}=\nabla \times \vec{A}$, we can define an effective magnetic field linked to the noncommutative parameter $\kappa$ via the relation

$$
B_{\kappa}=\frac{\partial A_{\kappa y}}{\partial x}-\frac{\partial A_{\kappa x}}{\partial y}=\frac{\kappa}{\hbar}
$$

which has different consequences. For instance it can be used to propose a new way to realize the quantum Hall effect without need of a real magnetic field. In addition, (8) allows to determine the solutions of the energy spectrum in similar way to that of the Landau problem in two-dimensions.

The Hamiltonian $H_{\kappa}$ can be diagonalized by considering the complex notation such that $z=x+i y$ and $2 p_{z, \bar{z}}=p_{x} \mp i p_{y}$. Then, after some algebra, we obtain

$$
H_{\kappa}=v_{\mathrm{F}}\left(\begin{array}{cc}
0 & 2 p_{z}+i \frac{\kappa}{2 \hbar} \bar{z} \\
2 p_{\bar{z}}-i \frac{\kappa}{2 \hbar} z & 0
\end{array}\right) .
$$

Such form suggests to linearly recombine the complex variables in order to realize the bosonic ladder operators of the harmonic oscillator. They are given by

$$
a=\frac{1}{\sqrt{2 \kappa}}\left(2 p_{\bar{z}}-i \frac{\kappa}{2 \hbar} z\right), \quad a^{\dagger}=\frac{1}{\sqrt{2 \kappa}}\left(2 p_{z}+i \frac{\kappa}{2 \hbar} \bar{z}\right)
$$

which satisfy the commutation relation $\left[a, a^{\dagger}\right]=\mathbb{I}$. Then, the Hamiltonian can easily be mapped in terms of such operators as

$$
H_{\kappa}=v_{\mathrm{F}} \sqrt{2 \kappa}\left(\begin{array}{cc}
0 & \hat{a}^{\dagger} \\
\hat{a} & 0
\end{array}\right) .
$$

Therefore, the solutions of the energy spectrum can be obtained using the number operator $N=a^{\dagger} a$ together with the harmonic oscillator states. Indeed, we solve the eigenvalue equation $H_{\kappa} \psi=E \psi$ to end up with the quantized energy in terms of the noncommutative parameter $\kappa$

$$
E_{\kappa, n}= \pm v_{\mathrm{F}} \sqrt{2 \kappa n}, \quad n \in \mathbb{N}
$$

exhibiting effective Landau levels in similar way to that of the Hamiltonian (2). This is showing an effective zero-energy Landau level, then one may talk here also about the consequence of the AtiyahSinger index theorem as has been done in [11]. The corresponding eigenspinors of (12) can be worked out using the condition $a \psi_{0}=0$ to obtain the degenerate groundstate

$$
\psi_{0}(z, \bar{z})=\sqrt{\frac{\kappa}{2 \pi \hbar}} e^{-\frac{\kappa}{4 \hbar} z \bar{z}}
$$

and to get the full state we need to define another set of bosonic operators commuting with the former ones. They can be written as

$$
b^{\dagger}=\frac{1}{\sqrt{2 \kappa}}\left(2 p_{\bar{z}}+i \frac{\kappa}{2 \hbar} z\right), \quad b=\frac{1}{\sqrt{2 \kappa}}\left(2 p_{z}-i \frac{\kappa}{2 \hbar} \bar{z}\right)
$$

and verify $\left[b, b^{\dagger}\right]=\mathbb{I}$. Calculating the action of the operator $\left(b^{\dagger}\right)^{m}$ on $(26)$, we find the normalized wavefunction

$$
\psi_{0, m}(z, \bar{z})=\sqrt{\frac{\hbar}{\pi \kappa 2^{m+1} m !}}\left(\frac{\kappa}{\hbar} z\right)^{m} e^{-\frac{\kappa}{4 \hbar} z \bar{z}}, \quad m \in \mathbb{Z}
$$


and therefore the action $\left(a^{\dagger}\right)^{n} \psi_{0, m}(z, \bar{z})$ gives the normalized eigenspinors of the Hamiltonian $H_{\kappa}$

$$
\Psi_{n \neq 0, m}=\left(\begin{array}{c}
\psi_{n, m} \\
\psi_{n-1, m}
\end{array}\right)
$$

where the eigenfunctions $\psi_{n, m}$ take the form

$$
\psi_{n, m}(z, \bar{z})=\sqrt{\frac{\hbar n !}{\pi \kappa 2^{m+1}(n+m) !}}\left(\frac{\kappa}{\hbar} z\right)^{m} L_{n}^{m}\left(\frac{\kappa}{2 \hbar} z \bar{z}\right) e^{-\frac{\kappa}{4 \hbar} z \bar{z}} .
$$

Note that, the zero-mode eigenspinors is

$$
\Psi_{0, m}=\left(\begin{array}{c}
\psi_{0, m} \\
0
\end{array}\right)
$$

To close this part, we notice that by switching off $\kappa$ we recover the linear dispersion relation and the associated eigenspinor. Additionally, the solutions of the energy spectrum for a real magnetic field can be obtained using the identification settled in (8).

\section{Measurement of $\kappa$}

Graphene has an hexagonal structure with is one-atom-thick film, which can be considered as a flexible membrane. With such characteristics, the connection between its mechanical and electronic properties is possible. Indeed, the interplay between its elastic and electronic properties was investigated in different occasions [3-5]. Experimental results showed that the graphene nanobubble under strain effect leads to a huge pseudomagnetic field ( $300 \mathrm{~T}$ ), which has never been created in the laboratory [16]. Then, a uniaxial strain larger than $23 \%$ in the zigzag direction can generate a transport gap in the transmission $[17,18]$. Additionally, the mechanical strains in graphene can also shift the Dirac points and generate new ones, which push Dirac fermions to have asymmetrical velocity $v_{x} \neq v_{y}$ along $x$ - and $y$-directions [19]. Motivated by the above results, we propose a way to measure the noncommutative parameter $\kappa$ by considering graphene under constraints.

To clarify the above statement, we introduce the strain tensor for a given a spatially varying displacement field $\vec{u}=u_{x} \vec{e}_{x}+u_{y} \vec{e}_{y}+h \vec{e}_{z}$ in cartisian coordinates with $u_{i}$ and $h$ are the in and out of plane deformations. Such tensor is given by

$$
\varepsilon_{i j}=\frac{1}{2}\left(\partial_{j} u_{i}+\partial_{i} u_{j}+\partial_{i} h \partial_{j} h\right), \quad i, j=x, y
$$

and then the structural distortion-induced strain on graphene can be evaluated using the following components

$$
\begin{aligned}
& u_{x x}=\frac{\partial u_{x}}{\partial x}+\frac{1}{2}\left(\frac{\partial h}{\partial x}\right)^{2}, \quad u_{y y}=\frac{\partial u_{y}}{\partial y}+\frac{1}{2}\left(\frac{\partial h}{\partial y}\right)^{2} \\
& u_{x y}=\frac{1}{2}\left(\frac{\partial u_{x}}{\partial x}+\frac{\partial u_{y}}{\partial y}\right)+\frac{1}{2}\left(\frac{\partial h}{\partial x}+\frac{\partial h}{\partial y}\right) .
\end{aligned}
$$

To explicitly determine these components, we work out with some requirements related to graphene under strain effect. Indeed, let us choose the $x$-direction such that the structure deformation leads to 
an effective gauge $\vec{A}_{\kappa}=\frac{\kappa}{2 \hbar}(x, y)$ associated with the shear deformation at one site (in the first order approximation) $[20,21]$

$$
\begin{aligned}
& \frac{\kappa}{2 \hbar} y= \pm \frac{c \beta}{a}\left(u_{x x}-u_{y y}\right) \\
& -\frac{\kappa}{2 \hbar} x=\mp 2 \frac{c \beta}{a} u_{x y}
\end{aligned}
$$

where $\beta=-\partial \log t / \partial \log a$ is the electron Grüneisen parameter, $a$ is the lattice spacing, $t$ is the electron hopping between $p_{z}$ orbitals located at nearest neighbor atoms, $c$ is a numerical constant that depends on the details of atomic displacements within the lattice unit cell, the signs \pm refer to the $K$ and $K$ valleys, respectively. (24) and (25) show clearly the mapping between the noncommutativity produced by the coordinates and deformation induced in graphene under the strain effect. Impotently, the mapping presents an alternative way to give prominence to the experimental realization of the noncommutativity.

On the other hand, according to reference [4] it is convenient to study the stress tensor for the elasticity problems in two-dimensions. Then for a given elastic energy $\mathcal{F}$ such tensor can be defined as $\sigma_{i j}=\partial \mathcal{F} / \partial u_{i j}$. Since we have defined an effective gauge associate to the noncommutative parameter $\kappa$, then immediately one can set the relations

$$
\begin{aligned}
& \frac{\kappa}{2 \hbar} y= \pm \frac{c \beta}{2 a \mu}\left(\sigma_{x x}-\sigma_{y y}\right) \\
& -\frac{\kappa}{2 \hbar} x=\mp 2 \frac{c \beta}{a \mu} \sigma_{x y}
\end{aligned}
$$

and $\mu$ is the Lam coefficient. Under some conditions, it was shown that the elastic energy has a cubic dependence on the coordinates, such as [4]

$$
\mathcal{F}(x, y)=c_{1}(x+i y)^{3}+c_{2}(x-i y)(x+i y)^{2}
$$

where $c_{1}$ and $c_{2}$ are two arbitrary constants. Note that (26) can be worked out to obtain four possible functions that result in uniform pseudomagnetic field. Then among them, we choose the following

$$
\sigma(x, y)=\sigma_{0}\left(3 x^{2} y-y^{3}\right)
$$

with $\sigma_{0}$ is a constant depending on the applied forces. For the valley $K$, we evaluate (24) and (25) to establish a link between $\kappa$ and $\sigma_{0}$

$$
\kappa=\left(12 \frac{c \beta \hbar}{a \mu}\right) \sigma_{0}
$$

To give an explicit value of $\kappa$ as function of $\sigma_{0}$ in the case of graphene, we consider the physical parameters $\beta \approx 2, a=0.142 \mathrm{~nm}, t=3 \mathrm{eV}, c=1, \mu=9.4 \mathrm{eV}^{-2}$. By replacing in (28) we offer a measurement of the noncommutative parameter based on the values taken by $\sigma_{0}$. Then, in units $\hbar / e=1$, we end up with

$$
\kappa=\left(\begin{array}{lll}
0.11836412 & 10^{-24} \sigma_{0}
\end{array}\right) \mathrm{m} \mathrm{s}
$$

where $\sigma_{0}$ has unit $\mathrm{N} / \mathrm{m}^{2}$. This result may give a hint how to experimentally fix the noncommutative parameter and therefore offer a measurement of it. Moreover, we can use other solutions rather than (27) to generate other form of $\kappa$ depending on the geometry of the graphene system. 


\section{Conclusion}

We have proposed a method to measure noncommutativity of the phase space variables using the properties of graphene under the strain effect. More precisely, we have considered Dirac fermions moving in the noncommutative plane and built up a Hamiltonian similar to that describing the same system but experiencing a real perpendicular magnetic field. This similarity allowed us to define an effective gauge and obtain the effective Landau levels measured in terms of the noncommutative parameter $\kappa$ resulting from the noncommuting momenta.

We have linked the noncommutativity features to two effects. Indeed, firstly we have interpreted $\kappa$ as a manifestation of an effective magnetic field generated by folding space and giving rise to a quantized energy. Secondly, by analogy to pseudovector potential, we have defined $\kappa$ as function of the strain tensor components. To give an explicit form of $\kappa$ in terms of the physical parameters, we have considered the stress tensor and by fixing the physical parameters in the case of graphene, we were be able to give numerical values of $\kappa$ as function of the stress constant $\sigma_{o}$.

\section{Acknowledgment}

The generous support provided by the Saudi Center for Theoretical Physics (SCTP) is highly appreciated by AJ.

\section{References}

[1] K. S. Novoselov, A. K. Geim, S. V. Morozov, D. Jiang, Y. Zhang, S. V. Dubonos, I. V. Gregorieva, A. A. Firsov, Science 306, 666 (2004).

[2] A. H. Castro Neto, F. Guinea, N. M. R. Peres, K. S. Novoselov, A. K. Geim, The electronic properties of graphene. Rev. Mod. Phys. 81, 109 (2009).

[3] M. A. Vozmediano, M. Katsnelson, and F. Guinea, Physics Reports 496, 109 (2010).

[4] F. Guinea, A. K. Geim, M. I. Katsnelson, and K. S. Novoselov, Phys. Rev. B 81, 035408 (2010).

[5] M. Van Wijk, A. Schuring, M. Katsnelson, and A. Fasolino, 2D Materials 2, 034010 (2015).

[6] F. Guinea, M. I. Katsnelson, and A. K. Geim, Nat. Phys. 6, 30 (2009).

[7] P. Nigge, A. C. Qu, É. Lantagne-Hurtubise, E. Mrsell, S. Link, G. Tom, M. Zonno, M. Michiardi, M. Schneider, S. Zhdanovich, G. Levy, U. Starke, C. Gutirrez, D. Bonn, S. A. Burke, M. Franz and A. Damascelli, Sci. Adv. 5, 5593 (2019).

[8] K. S. Novoselov, A. K. Greim, S. V. Morosov, D. Jiang, M. I. Katsnelson, V. I. Grigorieva, L. Levy, S. V. Dubonos, A. A. Firsov, Nature 438, 197 (2005).

[9] Y. Zhang, Y. W. Tan, H.L. Strmer, P. Kim, Nature 438, 201 (2005).

[10] M. F. Atiyah and I. M. Singer, Ann. Math. 87, 484 (1968); Proc. Natl Acad. Sci. USA, 81, 2597 (1984). 
[11] M. I. Katsnelson, Graphene: Carbon in Two Dimensions (Cambridge University Press, 2012).

[12] A. Connes, Noncommutative Geometry (Academic Press 1994).

[13] O. F. Dayi and A. Jellal, J. Math. Phys. 43, 4592 (2002).

[14] A. Jellal, J. Phys. A: Math. Gen. 34, 10159 (2001).

[15] A. Jellal and R. Houça, Int. J. Geom. Meth. Mod. Phys. 6, 343 (2009).

[16] N. Levy, S.A. Burke, K. L. Meaker, M. Panlasigui, A. Zettl, F. Guinea, A. H. Castro Neto, M. F. Crommie, Science 329, 544 (2010).

[17] M.M. Fogler, F. Guinea, M.I. Katsnelson, Phys. Rev. Lett. 101 (2008) 226804.

[18] V. M. Pereira and A. H. Castro Neto, Phys. Rev. Lett. 103, 046801 (2009).

[19] S.-M. Choi, S.-H. Jhi, Y.-W. Son, Phys. Rev. B 81, 081407(R) (2010).

[20] H. Suzuura and T. Ando, Phys. Rev. B 65, 235412 (2002).

[21] J. L. Maes, Phys. Rev. B 76, 045430 (2007). 